\title{
Comparative Research on the Importance and Performance of Instructor Pilots' Core Competencies
}

\author{
Kyu Wang Kim ${ }^{1}$, Kang-Seok Lee ${ }^{2^{*}}$ \\ ${ }^{I}$ Department of Flight Operation, Hanseo University, South Korea \\ ${ }^{2}$ Department of Air Transport \& Logistics, Hanseo University, South Korea
}

*Corresponding Author: Kang-Seok Lee, Department of Air Transport, Hanseo University, South Korea

\begin{abstract}
Through the study, we found current civilian airline instructor pilots' leadership core competencies and developed sub-activity indicators to analyze the differences in how instructor pilots are actually performing. Through literature study, we selected leadership core competencies and developed behavior indicators to measure the selected core competencies. With 19 core competencies and 75 behavior indicators developed initially, we surveyed pilots currently working in the field in terms of importance. Using the results, we eliminated behavior indicators that our expert group deemed ambiguous and those scored less than 4.0 in importance. Through this procedure, we selected 19 core competencies and 59 behavior indicators. As a result of analyzing core competencies and reliability, it was shown that instructor pilots' leadership core competencies and sub-activity indicators were limited but reliable. We analyzed each core competencies' importance according to their competency models. Among the core competencies, 9 of them including working training, type of license, and where their career started were shown to be insignificant. Morality and responsibility among personal attributes/traits showed significant differences. We compared what pilots deem important and how they actually perform at workplace based on actually developed measures. Comparative analysis showed relatively large differences between the importance and the performance
\end{abstract}

\section{INTRODUCTION}

Transportation, which was mostly carried out by land transportation in the past, is moving to the sky in large part due to recent technological developments in aviation. The passenger transportation revenue of Korean airlines has increased by about 529\% in 2017 compared to 1990. Similarly, the air cargo revenue has increased by about $450 \%$ in 2017 compared to 1990, growing steadily at an annual rate of $9.29 \%$ and $8.39 \%$, respectively (Ministry of Land, Infrastructure and Transport, Aviation Statistics, 2017). In addition, new budget airlines are expanding with business license authorization, and the required number of pilots is increasing accordingly. According to the Korea Aviation Association's 2016 Aviation Yearbook, the average e annual growth rate for the number of pilots is $6.2 \%$ during ' $04 \sim ' 16$ and about $5.8 \%$ for the past five years. As of 2016, a total of 5,667 pilots are operating in 9 national airlines (Korean Air, Asiana Airlines, Jeju Air, Jin Air, Air Busan, Eastar, Tway Air, Air Incheon, Air Seoul). Therefore, both quantitative and qualitative growth of manpower is necessary for safe air transportation. Putting aside the problem of increasing the number of pilots and focusing only on qualitative growth, the role and responsibility of instructor pilots are crucial as they are at the forefront of producing pilot manpower. Therefore, it is essential to know what the key factors are for instructor pilots who are directly involved in improving the quality of pilot manpower. Effective management requires sound evidence and research. Therefore, this study was conducted to establish a standard for the management of instructor pilots as they nurture pilots who are primarily responsible for safe air transportation.

This study classifies and defines common behavioral characteristics found among outstanding instructor pilots in civil airlines. It also proposes how to manage instructor pilots within the training system.

In education, the correspondence in thinking between educators and trainees is fundamental, and the result of education is greatly influenced accordingly. Therefore, it is necessary to analyze the degree of agreement between instructors' education and their trainees' understanding in the actual flight education. In order to confirm this, we investigated the perceived importance of instructor pilots' core competency by general pilots and their actual performance. 


\section{THEORETICAL BACKGROUND}

\subsection{Instructor's Job Characteristics}

According to the pilots' duties under the Aviation Safety Act, it is necessary to have the knowledge and skills to protect property and life with safety as the top priority during transportation of passengers and cargo by aircraft (Article 1 Purpose of the Aviation Safety Act; authority). FAA-H-8083-9A (Aviation Instructor's Handbook), published by the Federal Aviation Administration (FAA), provides instructor pilots with up-to-date information on learning, teaching and how to implement aviation and aeronautics education. Based on this, the duties of a desirable instructor pilot can be summarized as follows. Instructor pilots should be able to apply individualized training to student pilots with different individual characteristics based on their understanding of human behavior and student pilots' defense mechanisms. They must have respectable traits so that student pilots can voluntarily follow, and they should also have the knowledge and skills to fly safely. They should be able to communicate the knowledge and skills effectively and practice with the willingness to follow regulations and procedures. They should encourage student pilots to drive efforts to develop their own potential.

\subsection{Concept of Core Competencies}

\subsubsection{Previous Research on Leadership}

In general, leadership is a common term that is often referred to in working environments of everyday organizations, but many scholars define it in terms of academic and professional level. Stogdill, R. M. (1974) said "There are as many different leadership definitions as there are scholars who have tried to define it." Implying that leadership has a lot of meanings and complexity. Different scholars define leadership differently in terms of individual qualities, behavioral characteristics, achievement of organizational goals, and how their influence on the behavior and attitudes of followers following leaders is exercised and deployed.

\subsubsection{Instructor Pilot Leadership}

The concept and definition of leadership has not changed fundamentally, except that it reflects the times from the past to the present. The ultimate quest for leadership is to induce subordinates to voluntarily follow their leaders. In a comprehensive viewpoint, a leader properly exercises leadership in many aspects in life to induce actions. In order to make decisions and actions in an individual's life, one needs a principle that acts like a compass to give direction. Steven Kobe said in the principlebased leadership, leadership begins internally and moves outwards in four dimensions. The four dimensions are: 1) the personal level (relationship with oneself), 2) the interpersonal level (relationship and interaction with others), and 3) the management level (responsibility to complete work with others). 4) the organizational level (to bring people together, to educate, to pay, to form teams, to solve problems, and to align structural strategies and systems in one direction) (Stephen R. Covey, 2001). Civil aviation instructor pilots act as educators to help student pilots adapt to airlines and ensure safe flight. Therefore, the leadership exerted by instructor pilots can be seen as a sequential process of how the role of educator is exercised in four dimensions. The leadership of civil airline instructor pilots can be defined as: "Instructor pilots are encouraged to teach student pilots knowledge, skills and culture of flight, and to foster the potential of student pilots to help them grow themselves. They should be able to apply the four dimensions of leadership sequentially and enable student pilots to actively participate in achieving goals by inducing obedience to follow."

\subsubsection{Leadership Capabilities and Core Competencies}

Competencies are internal characteristics of an individual that contributes to effective and outstanding performance in compliance with a particular situation or job (Lyli M. Spenser, Jr., PhD Signe M. Spencer, 1998). Internal characteristics are in-depth and lasting aspects of an individual's personality that enable one to predict the individual's behavior in various situations. What competencies contribute to performance means is that competencies are the causes of behavior or performance, and therefore behavior and performance can be predicted. To follow a standard means that competencies predict a person's excellence or impotence by specific criteria or standards. Competencies as internal characteristics of an individual can be described by dividing it into five types (Lyli M. Spenser, Jr., PhD Signe M. Spencer, 1998).

Among the many concepts of competency, there are three characteristics that appear in common. First, there is an emphasis on connection within work achievement and performance in common, and 
competency must be directly related to performance ability that can produce results. Second, although there are differences among researchers, competency is usually defined as the knowledge area that are objectively acquired, the skill area that deal with work techniques and procedures, and the attitude area that relate to personal characteristics and motivation. Third, it should be expressed as criteria that can be observed and measured. This is mainly defined as the concept of performance behavior (Lee Hong-min, Kim Jong-in, 2006). In the leadership perspective, competency can be understood as a collection of knowledge, skills, and attitude areas that a leader should possess and performance that can be observed and measured. Therefore, the instructor's leadership competence in this study can be defined as the ability to teach student pilots and foster their potential to grow on their own by using the individual's knowledge, skills, and internal traits within the personal, interpersonal, management, organizational levels in a sequential manner, resulting in the student pilot's voluntary obedience, and it is internal characteristics of the individual that can be observed and measured objectively. Core competency refers to the collective capabilities, skills, and knowledge that are unique to a company that have been leading the company in the past and able to be transformed appropriately to accumulate additional capabilities and drive future growth. It is a comprehensive competency that includes the know-how, technology or simple functions unique within the corporation that others cannot imitate, and hence becomes the source of the company's differentiated competitiveness. Leadership core competencies are 'the core competencies, among other competencies required as leaders, including the unique ability, skills, and knowledge that can distinguish between good and poor leaders'.

\subsection{Leadership Core Competency Modeling}

Most competency modeling procedures and methods are rooted in McClelland's Job Competence Assessment Method (JCAM). Phase 1 examines job components (positional tasks, job activities, roles, organizational environment and issues) and the requirements of outstanding job performers. First of all, a focus group is set up for the job experts, excellent job performers, job managers, and managers to extract the personal characteristics of the good performers. The ranking is then estimated according to the importance of the performance to estimate the individual characteristic ranking list. It also discreetly selects excellent job performers. Phase 2 examines the characteristics of outstanding job performers and constructs a job competency model. Job observations and key behavioral interviews are conducted one-on-one for high performers and average performers to form competency models that identify competencies that are unique to good performers. The model consists of three steps. Measure the reliability and validity of the model's contents, and as a necessary step to increase effectiveness, it should be approved by the organization's management and key leaders. In the verification process, the method of repeating the initial study and using an external consultant as a panel are frequently used (Lee Hong-min and Kim Jong-in, 2006). As discussed in the abovementioned research perspectives and development methods for competency, there are many ways to develop a competency model for an organization, which can be selected and utilized according to the intention of the researcher.

\subsection{Role and Core Competencies Required for Instructor Pilots}

Just as the needed leadership has changed with time, the type of leadership that instructor pilots are required to have has also changed. If instructor pilots were trained based on control and command in the past, leadership should be exercised based on the instructor's capabilities from now on. When considering leadership based on competency, instructor pilots need to be wise and versatile. Stephen Kobe's principle-driven leadership is said to begin from the inside and work out in four dimensions. The four dimensions are personal, interpersonal, managerial and organizational (Stephen R. Covey, 2001). The four dimensions of leadership and the internal characteristics can be differentiated by dividing the personal dimension into the self-concept, traits, and motivation at the interpersonal relationship, management, and organizational level. Therefore, in this study, self-concepts, traits, and motivations were classified as basic qualities at the personal level, and the competencies needed at the interpersonal, management, and organizational dimensions were divided into two categories. Competencies in the interpersonal, management, and organizational dimensions included in the organizational management level were each classified into one big competency group, which is divided into interpersonal, task performance, and organizational management competencies.

Therefore, the four competency groups of the instructor pilot leadership core competencies are personal attributes/traits, interpersonal, work performance and organizational management. Personal 
attributes/traits reflect a consistent responsiveness and attitude, value, or self-image to motives, circumstances, or information that cause the individual to be consistently mindful or desired (Stephen R. Covey, 2001). Interpersonal competency is related to the development of the potential of members (student pilots) who are responsible for achieving organizational goals and producing results, and inducing an active attitude toward work performance. Work performance competencies are those related to the efficient and effective performance of daily tasks (flight training) or related tasks to achieve organizational goals. Finally, organizational management competencies are those related to aligning members in one direction to achieve the organizational goals assigned to the organization as members and managers (Byung-Soon Choi, 2007).

The role of instructor pilots extends from the individual level to the interpersonal, work performance, organizational level, and it is more extensive than ever and requires an integrated approach. In addition, the critical competencies for the required roles should be more specific and more practically researched.

\section{RESEARCH METHOD}

\subsection{Selecting Leadership Core Competencies and Developing Behavioral Indicators}

Competency modeling was integrated eclectically with behavioral event interviews, expert panel participation, job function analysis, and the use of competency dictionaries.

The core behavioral interview was conducted in a 1:1 interview with 95 pilots from airline $\mathrm{K}$ and airline A (average 15 years and 8 months of service) who are currently in civilian flight duty.

The panel of experts consisted of instructor team leader, inspector group leader and quality control team leader currently working at $\mathrm{K}$ airline to explore the characteristics found in pilots who are evaluated as competent instructors.

In addition, airline K and airline A's Sustainability Report, airline K's LCPM (Line Check Pilot Manual), OE (Operating Experience) Critique Form, and Behavioral Indicator used by CRM (Crew Resource Management) Team, FAA-H-8083-9A (Aviation Instructor's Handbook) were used to analyze the instructor's function. Additionally, the competency dictionary included in the technical book was used.

The whole process was carried out compositely. The interview asked three questions: What should the instructor pilot teach the student pilot, what do you think the role of the instructor pilot is, and which instructor is a good instructor pilot? Afterwards, the responses were summarized and linked to each of the competencies.

Many of the details of the instructor's behavior were elaborated, so the data were organized separately for use as behavioral indicators. The reviewed contents were rearranged by a group of experts and finally 19 were selected from 25 .

'Honorship' was reclassified as 'Honesty' and 'Morality' because it was deemed inappropriate to apply to a group of civilian pilots. In addition, in order to apply the meaning of the general "leader model" to the pilot in detail, the term was changed to "perform standardization procedure". Since the 'delegation of authority' taking place in the cockpit is deputing the control of the flight, it was modified to be a 'control delegation' to reflect this. Since "insight" can be categorized as work expertise by understanding the overall situation of flight, the action indicator corresponding to insight is integrated into 'professionalism'.

19 competencies and 75 behavioral indicators have been developed to define the core competencies derived and the behavior observed in the performance of competent instructor pilots. The developed behavioral indicators include 3 in honesty, 3 in morality, 1 in fairness, 1 in responsibility, 1 in confidence, 1 in passion, and 4 in self-regulation, totaling 14 in terms of personal attributes/traits

A total of 17 behavioral indicators were developed in terms of interpersonal competence, with 7 in understanding human behavior, 2 in respect for others and 8 in relationship formation.

3 in standardization procedures, 6 in communication, 9 in control delegation, 3 in motivation, 3 in risk management, 3 in principle-based consistency, 8 in professionalism, and a total of 36 behavioral indicators were developed in terms of work performance capacity.

Total of 9 behavioral indicators were developed in organizational management competency by developing 6 behavioral indicators in positive culture creation and 3 in goal setting. 


\subsection{Survey}

The survey was conducted for pilots working for airline K and airline A over 4 weeks from September 10 to October 5, 2018. A total of 243 people responded to the survey with 23 instructor/censor groups representing $9.5 \%, 115$ captains representing $47.5 \%$, and $43.5 \%$ for 105 co-captains.

In order to confirm the integrity of the survey, 2 inverse questions were included in the total of 77 questions. According to the degree of importance of the 5-point Likert scale, 'not important $=1$ point', 'not very important $=2$ points', 'normal $=3$ points', 'a bit important $=4$ points', 'very important' $=5$ points. For obscure questions that were not clear or appropriate were given 22 ". Question 36 is the inverse of question 6, 'Respect the student pilot's opinion.' Question 70 is the inverse of question 13, 'Does not criticize or get angry.'

\section{RESEARCH METHOD}

Among the 243 people who participated in the questionnaire, 31 answers were insincere, so those $13 \%$ of the surveys were dropped, and 212 , or $87 \%$ of the survey responses, were analyzed.

The survey was conducted with 19 instructor/censor pilots, 98 captains and 95 deputy captains, totaling 212 participants. In fact, the satisfactory response rate was $82.6 \%$ for instructors/censor pilots, $85.2 \%$ for captains, and $90.5 \%$ for co-captains. By the number of working years of participants, $27.9 \%$ was under 5 years, $36 \%$ was $6-10$ years, $68 \%$ was $11-20$ years, and $49 \%$ was 21 years or more. The respondents represented a wide range of distribution, and it can be justified that the data obtained is relatively objective. The survey result had 9 aircraft categories, but when classifying by aircraft category, there were big differences in the number of participants from 2 to 86 . Therefore, with the advice of a group of experts, we decided to exclude the analysis by model. Among the 212 questionnaires, 90 responses, $42.5 \%$ of the data, were military career originated and 122 responses, $57.5 \%$, were of the civilian history, which are relatively similar participation rates.

After discussing with a group of experts, it was decided to drop the behavioral indicators below 4.0. This was because 4 points can be assumed to be 80 points in terms of percentage, and it was determined that this score is acceptable. On the other hand, the more complex the behavioral indicators are, the more relevant the core competencies can be analyzed.

More than nine respondents of the 212 who responded sincerely, responded that question 67 was inadequate, so the question was dropped through discussions with expert groups. This is because what behavior indicators evaluate or what they mean is not accurately identified. The finally selected behavioral indicators were 59 after dropping 16. Excluding the importance and inappropriate behavioral indicators, 7 of the 17 behavioral indicators were dropped from interpersonal competency, and only 10 behavioral indicators were adopted. The drop rate is $41.2 \%$. In the Organizational Management Competency Group, four of the nine behavioral indicators were eliminated and only five behavioral indicators were adopted. The drop rate is $44.4 \%$. Personal attributes/traits were not eliminated. This shows that general pilots consider the instructors' personal attributes/traits to be very important. Work performance is also important in teaching flight. The fact that the pilots' organization has somewhat different characteristics from other organizations can be confirmed by the fact that various behavioral indicators have been dropped from the interpersonal and organizational management capabilities.

\subsection{Validation of the Validity And Reliability of Behavioral Indicators}

The validity and reliability were verified through factor analysis of the final 59 behavioral indicators adopted. Among exploratory factor analysis, principal component analysis was applied to verify validity. Among the orthogonal factor rotation methods, the factor was extracted using the Varimax Rotation method, and as a result, factors were separated into 11 dimensions.

As a result of factor analysis, 19 out of 59 behavioral indicators were dropped and only 40 behavioral indicators were selected, and only 11 factors were derived. Based on the results, the core competency and behavioral indicators were revised again with advices from a group of experts.

It is the operational definition of the finally selected core competency. Honesty is the attitude of accepting one's mistakes and maintaining it without falsehood or embellishment. Morality is the attitude of respecting others and not boasting. Confidence is a personal feeling of being able to do 
something well. Understanding human behavior is the ability to systematically understand, predict, and control human behavior. Respect for others is the attitude to treat others with honor. Implementing standardization procedures is an act of setting an example for flight standardization and trying to follow rules and procedures. Delegation of control is an act of giving student pilots the ability to make direct judgment and control by providing effective and appropriate information to student pilots. Motivation is an act of encouraging behavior by stimulating and encouraging the causes that cause people to act. Risk management is the ability to deal with a crisis calmly even when facing an emergency. Cooperation is the ability to work in collaboration with student pilots or to work as a team member to achieve common goals. Creating a safety culture is the ability to create and manage a safety culture so that student pilots share pride and desirable organizational values.

Reliability test by internal consistency (Cronbach $\alpha$ ) was performed. Based on the survey results, the instructor pilot's core competencies and behavioral indicators for each competency are valid and reliable in descriptive statistics.

According to the factors, the reliability coefficient was .744 for personal attribute/trait, .761 for interpersonal relationship, .932 for work performance, and .864 for organizational management. In particular, work performance can be judged as the most reliable classification.

\subsection{Importance and Performance Analysis}

\subsubsection{Importance}

One particular importance is that one of the core competencies belonging to the upper group are related to personal attributes/traits work and performance.

\subsubsection{Performance}

The participant pilots were asked to evaluate their instructor pilots in their past $\mathrm{OE}($ Operating Experience) in terms of performance.

The performance deviation remains as small as .65 to as large as .90. 'Honesty' and 'respect for others' can be regarded as competencies that vary greatly by the instructor pilot.

Confidence and risk management, on the other hand, are competencies that differ in small degrees. Therefore, it may be more efficient for the manager in charge to focus on competencies with low deviation and average values.

\subsubsection{Importance and Performance by Competency}

The cooperative competency is the biggest difference. Cooperation is the ability to work in collaboration with student pilots or to work as a team member to achieve common goals. There are five behavioral indicators that make up cooperativeness.

In this study, the core competencies of performance compared to importance were honesty (0.94), respect for others (0.73), control (0.73), and safety culture (0.72). In particular, there was a significant result between civil and military careers in responsibility and morality, and the remaining behavioral indicators indicated that there is a general consensus in the results as the results were not significant regarding the unique pilot occupation. In addition, the differences in importance and performance in the core competencies of confidence and risk management were relatively smaller than those of other competencies, 0.38 and 0.55 , respectively. The significance probability ( $\mathrm{p}$ value) of less than 0.05 in all core competencies indicates that the results of the difference analysis of importance and performance are very significant.

\section{CONCLUSiON}

This study was conducted to find out the leadership core competencies of civil aviation instructor pilot and develop sub-activity indicators of the core competencies, and to analyze the differences in how actual instructor pilots are performing. Through literature studies, we developed leadership indicators and behavioral indicators to identify selected core competencies through in-depth interviews, expert discussions, and literature and company K's evaluation forms. Based on the 19 developed core competencies and 75 behavioral indicators, the survey was conducted on the pilots working in the field. As a result of the survey, the behavioral indicators determined to be ambiguous and the behavioral indicators of importance less than 4.0 were excluded. Thus, 19 core competencies and 59 behavioral indicators were selected. In order to analyze the validity of the behavioral indicators in the core competency group, factor analysis was re-adjusted, and the core competency group was re-adjusted from 19 to 11, and the behavioral 
indicators were also re-adjusted to competencies tied to the same factors. Behavioral indicators were developed. Instructor pilots' core competencies were selected as three personal attributes/traits (honesty, morality, and confidence), and two subordinate indicators were in honesty, three in morality and three in confidence. At the organizational level, two interpersonal relationships (understanding of human behavior and respect for others) were selected, and three behavioral indicators were developed. Five tasks were selected (perform standardization procedures, mandates, motivation, risk management, coordination), three in standardization procedures, four in control, four in motivation, five in risk management, five in cooperation, totaling in 21 behavioral indicators being developed. Finally, one was developed in organizational management (creating a safety culture) and six sub-activity indicators. 11 core competencies and 40 behavioral indicators were developed. Analyzing the reliability of core competencies and behavioral indicators, the instructor pilot's core competency and subordinate behavioral indicators were limited in data but reliable. The importance of each capability was analyzed according to each core competency modeling. Nine of the core competencies were not significantly different by age, qualification or origin. Honesty and morality among personal attributes/traits showed significant differences between military and civil careers. Based on the actual developed contents, the degree of analysis conducted on the job site and the degree judged to be important were analyzed. As a result of the analysis, the difference in importance and performance was found to be remarkable.

\subsection{Limitations of Research}

This study has a lot of significance in the present study of civil aviation instructor pilot, but also has the following limitations.

First, additional research in the development of civil aviation instructor pilot core competency and behavioral indicators is necessary through more precise revision of study and accumulation and verification of the long-term data. Although the study is valuable in its scarcity, it would be difficult to overcome the limitations of the one-time study unless further research is done for this reason.

Second, in order to investigate and analyze detailed organizational diagnosis and function by understanding the duties and organizational characteristics of the instructor pilot, a large number of participants and time are required, but there were limited resources for the study.

Third, this study provides criteria for the core competencies of the civil aviation instructor pilot, but it does not provide a concrete method for diagnosis. In order to reflect the research results in development, it is necessary to continuously research and support various policies and measures in connection with the department in charge of the management of civil aviation instructor pilot.

In order to overcome the above limitations and to develop the human resources management for civil aviation pilots and establish a policy accordingly, the directions for future research and development are as follows.

\subsubsection{Instructor Selection}

Although the personnel management of the airline staff, especially the pilots, has been conducted, it is difficult to ensure that it was systematic and high-quality education (humanity and knowledge skills) was conducted by selecting instructors according to the company's contacts, academics, delays, and company loyalty. Since pilot education is a key factor for improving the future and safety of civil airlines, the qualitative and quantitative education is the key to aviation industry and human management by systematically establishing the core competency of instructor leadership. It is suggested to introduce a talent pool system as a complementary measure in selecting instructors. The talent pool system generally refers to the system of recruiting talents by selecting applicants when recruitment is required after forming a workforce bank.

Since personal attributes are difficult to determine accurately and cannot be known with certainty, many can be observed and identified by structuring a system that can accumulate that data over time. Therefore, it is proposed to structure as follows.

Survey pilots who have sufficient qualifications as instructors based on items pertaining to quarterly personal attributes (including questionnaires prepared by questionnaire) among those eligible for instructor experience of 500 hours or more than 1000 hours. Based on the results of the survey and the accumulated data, by selecting the top-ranked instructors, it is possible to recruit agents with excellent personal attributes that are difficult to identify. It is believed that this will contribute to the development of more safe and professional pilots. 


\subsubsection{Conservative Education}

By utilizing the developed leadership competency and behavioral indicators, the results of comparing the differences between importance and performance can be regularly analyzed and operated to maintain the instructor training course for instructor pilots. It would be also possible to implement a flight education system. In other words, the survey is conducted on the indicators of how instructors are actually conducting instructors on a quarterly or semi-annual basis, and the results are analyzed. It can be grasped overall. Based on these results, the administrative personnel who manage the pilots can draft and implement the customized repair procedures needed at each time.

\subsubsection{Future Use of Research Data}

By further systemizing the current core competencies and behavioral indicators to improve reliability and validity, we hope this study results in the improvement in leadership among instructors/censors, captains, co-captains, and all pilots and development of self and other diagnostic tools to evaluate themselves and others' leadership core competencies.

\section{ACKNOWLEDGEMENT}

The research reported in this study was granted by Industry of Academic Cooperation Foundation, Hanseo University

\section{REFERENCES}

[1] SungHo Ko, et al, The Practice of Social Research, 11th edition, Thomson, 2007

[2] Republic of Korea Air Force Headquarters, "Korea Airforce Pilot Personality Inventory" Development Report, 2008

[3] Republic of Korea National Law Information Center, Aviation Safety Act, 2018

[4] NamYeoung Kim, "Research on Military Officer's Leadership Competency Model Development," Daegu Catholic University, 2015

[5] YongJae Kim, "Development and Application of Airforce Instructor Pilots' Leadership Competency Indicator," Airforce University, 2009

[6] JinMo Kim, "Development of Competency-Centered Curriculum for Companies' Human Resource Development," The Journal of Vocational Education Research,Volume 20, Number 2, 2001

[7] HongJun Kim, "Development of Hierarchical Leadership Competency Model for Public Organizations," Hanyang University, 2017

[8] Korean Air, "Sustaining Excellence," 2017

[9] Korean Air, "CRM Behavioral Indicator," 2017

[10] TaeHoon Yeon, "Research on Leadership Competency Indicator for Naval Lieutenants," Korea National Defense University,"2006

[11] Asiana Airlines, "Sustainability Report 2016," 2016

[12] YoungJinJeong, "Research on Core Competency Model for Airforce Pilot Officers," Korea National Defense University,"2000

[13] ByeongSoon Choi, et al, "Development of Leadership Indicator for Airforce Squadron Battalion Commanders, Korea National Defense University Research Institute for National Security Affairs,2007

\section{AUTHORS' BIOGRAPHIES}

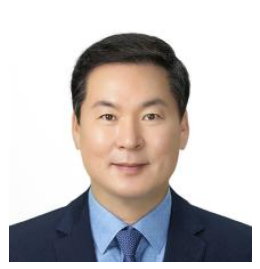

Kyu-Wang Kim, Adjunct Professor, Department of Flight Operation, Hanseo University, San 105, Sinon-ri, Nam- Myun, Taean-Gun, Chungcheongnam-do, South Korea was born in Chungnam Province in Korea, April 19, 1965. Educational background is Bachelor of Science(1991) from Korea Aerospace University, M.S (2019) from Hanseo University. Major field of study is Air Transportation and Administration. Research interest field is Airline Pilot training in Korea and Human Factors. He is currently the president of Airline Pilots Association of Korea.

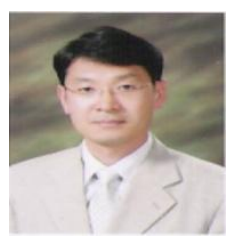

Kang-Seok Lee, Professor, Department of Air Transport \& Logistics, Hanseo University, San 105, Sinon-ri, Nam- Myun, Taean-Gun, Chungcheong nam-do, South Korea, was born in October 6, 1965. Educational background is Bachelor of Science (1991) of Aviation Management, M.S (1993) of Air Transport, Ph.D. of Air Transport (2000) degree from Korea Aerospace University in South Korea. Research interest field is Air Transportation related Airport Operation and Aviation Safety of Human 
Factors. Ph.D thesis is The Impact of Aviation Safety Over the Consumer's Behaviour and M.S thesis is A study on the Capacity Expansion for Kimpo International Airport. Professor, Lee is Member of ISASI (International Society of Air Safety Invesigators and ATRS (Air Transport Research Society). Also awarded Prime Minister Prize and Ministry of Land, Transport and Maritime Affairs Prize in $2005,2010$.

Citation: Kyu Wang Kim, Kang-Seok Lee. "Comparative Research on the Importance and Performance of Instructor Pilots' Core Competencies" International Journal of Managerial Studies and Research (IJMSR), vol 8, no. 11, 2020, pp. 1-9. doi: https://doi.org/10.20431/2349-0349.0811001.

Copyright: () 2020 Authors. This is an open-access article distributed under the terms of the Creative Commons Attribution License, which permits unrestricted use, distribution, and reproduction in any medium, provided the original author and source are credited. 